\title{
Comprehensive geriatric intervention program with and without weekly class-style exercise: research protocol of a cluster randomized controlled trial in Kyoto-Kameoka Study
}

This article was published in the following Dove Press journal:

Clinical Interventions in Aging

Yuya Watanabe, ${ }^{1-4, *}$ Yosuke

Yamada, ${ }^{2,4, *}$ Keiichi Yokoyama, ${ }^{2,5,6, *}$

Tsukasa Yoshida, ${ }^{2,4,7}$ Yasuko

Yoshinaka, ${ }^{5,6}$ Mie Yoshimoto, ${ }^{8}$

Yoko Tanaka, ${ }^{9}$ Aya Itoi, ${ }^{10}$ Emi

Yamagata, "' Naoyuki Ebine,'

Kazuko Ishikawa-Takata, ${ }^{12}$ Misaka

Kimura $^{2,3}$

'Faculty of Health and Sports Science, Doshisha University, Kyotanabe, Japan; ' 2 Laboratory of

Applied Health Sciences, Kyoto Prefectural

University of Medicine, Kyoto, Japan; ${ }^{3}$ Department

of Health and Sports Sciences, Kyoto Gakuen

University, Kameoka, Japan; ${ }^{4}$ Section of Healthy

Longevity Research, National Institute of Health

and Nutrition, National Institutes of Biomedical

Innovation, Health and Nutrition, Tokyo, Japan;

${ }^{5}$ Specified Non-profit Corporation Genki-up AGE

Project, Kameoka, Japan; ${ }^{6}$ Department of Business

Administration, Kyoto Gakuen University, Kyoto,

Japan; 'Senior Citizen's Welfare Section, Kameoka

City Government, Kameoka, Japan; ${ }^{8}$ Corporation

of Japan Dental Hygienists' Association, Kyoto,

Japan; ' Kyoto Dietetic Association, Kyoto, Japan;

${ }^{10}$ Department of Health, Sports and Nutrition,

Faculty of Health and Welfare, Kobe Women's

University, Kobe, Japan; '"Faculty of Nursing,

Doshisha Women's College of Liberal Arts,

Kyotanabe, Japan; '2 Department of Nutrition

and Metabolism, National Institute of Health

and Nutrition, National Institutes of Biomedical

Innovation, Health and Nutrition, Tokyo, Japan

*These authors contributed equally to this work

Correspondence: Misaka Kimura

Department of Health and Sports Sciences, Kyoto

Gakuen University, Kameoka, Japan, I-I Nanjo-Otani,

Sogabe, Kameoka, Kyoto 62I-8555, Japan

Email misaka@kyotogakuen.ac.jp

Yosuke Yamada

Section of Healthy Longevity Research, National

Institute of Health and Nutrition, National Institutes

of Biomedical Innovation, Health and Nutrition, I-23-I

Toyama, Shinjuku-ku, Tokyo I62-8636, Japan

Email yamaday@nibiohn.go.jp

\begin{abstract}
Objective: The number of long-term care (LTC) users and the associated expenditures in Japan are increasing dramatically. The national government recommends LTC prevention through activation of communities. However, there is no clear evidence of the effect of population-based comprehensive geriatric intervention program (CGIP) for restraints of LTC users and the associated expenditures in the future. The aims of the current paper are to describe the study protocol and progress of a cluster randomized controlled trial (RCT) with a CGIP in Kameoka City.
\end{abstract}

Methods: The cluster RCT involved random allocation of regions as intervention $(\mathrm{n}=4,859)$ and nonintervention $(n=7,195)$. Participants were elderly persons aged $\geq 65$ years without LTC certification who had responded to a mailing survey. The residents living in intervention regions were invited to a physical check-up, and 1,463 people participated (30.3\%). These individuals were invited to the CGIP, and 526 accepted. The CGIP comprised instructions on: 1) low-load resistance training using bodyweight, ankle weights, and elastic bands; 2 ) increasing daily physical activity; 3) oral motor exercise and care; and 4) a well-balanced diet based on a program from Ministry of Health, Labour and Welfare. We allocated the intervention regions randomly into home-based self-care program alone (HB group, 5 regions, $\mathrm{n}=275$ ) and home-based program+weekly class-style session (CS group, 5 regions, $\mathrm{n}=251$ ). We evaluated the effects of the CGIP at 12 weeks and at 12 or 15 months on physical function, and are conducting follow-up data collection for an indefinite period regarding LTC certification, medical costs, and mortality.

Results and discussion: The study was launched with good response rates in each phase. Participants of both groups significantly increased their step counts by $\sim 1,000$ per day from the baseline during the CGIP. This RCT will provide valuable information and evidence about effectiveness of a community-based CGIP.

Keywords: frailty, sarcopenia, long-term care, ActiveGuide, low-load resistance training, oral care, nutritional intervention, Shokuiku, physical function, physical activity monitor

\section{Introduction}

The Japanese Long-Term Care (LTC) Insurance System was launched in 2000, and revised in 2005 to implement care prevention measures. ${ }^{1}$ However, it is predicted that the country's burden of LTC care prevention will increase 5\%-6\% per year in Japan because of increases in the population and in the age of older adults in Japan. ${ }^{2}$ Therefore, the Integrated Community Care System was introduced as the LTC system's 2011 revision to restrain the cost of care prevention in LTC. In the system, 
local governments take some responsibility for providing a unique program to minimize the number of people certificated for LTC and to reduce the costs of providing LTC. ${ }^{2}$

The national government's Ministry of Health, Labour and Welfare (MHLW) recommends LTC prevention in the Integrated Community Care System through activation of communities in a multifaceted approach. ${ }^{3}$ MHLW recommends comprehensive geriatric interventions that comprise improvements in: 1) motor system function through exercise and physical activity; 2) oral function, through oral motor exercise and care; and 3) nutrition and diet, as well as problems associated with home boundness, such as social isolation, depression, and decreasing cognitive function. ${ }^{3}$ However, there is no clear evidence for population-based, comprehensive, longitudinal geriatric interventions to reduce burden on the LTC system in Japan.

There are potential resources in the form of experts and volunteers who are motivated to implement health promotion in communities; however, local governments are not in a position to manage them continuously. Therefore, there exists a need for a well-organized and long-term communitybased management team for preventing the need for LTC use. Such a team should include exercise experts, dietitians, dental care experts, the local medical network, local government employees, and community-dwelling volunteers who can participate at the neighborhood level. ${ }^{3}$ The communitybased comprehensive geriatric intervention program (CGIP) is required using these resources. Thus, we also established specified non-profit corporation, named Genki-up AGE Project, in Kameoka city and are continuously recruiting community-dwelling volunteers and training them as supporters of the program and neighborhood. This CGIP's design allows for sustainable expansion of the program to a large population in the future.

The study protocol has been carefully planned with city government, researchers, and healthcare providers; hence, it is too complex to be described in the context of a single outcome-focused article. The aims of the current paper are to describe the study protocol and to report the recruiting progress for a cluster randomized controlled trial (RCT) with a CGIP in the community.

\section{Methods}

\section{Study design, setting, and participants}

The cluster RCT was conducted in Kameoka City, Kyoto Prefecture, Japan. The entire study protocol was reviewed and approved by the Ethics Committee of Kyoto Prefectural University of Medicine (RBMR-E-371 and 372) and the
National Institute of Health and Nutrition (NIHN187-3). This study is registered with the University hospital's Medical Information Network Center - Clinical Trials Registration database (UMIN000008105).

Kameoka City is located in the central area of Kyoto Prefecture, about $25 \mathrm{~km}$ west of Kyoto City. The land area of the city is $224.90 \mathrm{~km}^{2}$. As of July 1, 2011, it had a population of 93,306 , with 19,424 (20.8\%) of individuals $\geq 65$ years. The city government conducted a postal survey of "Needs in the Sphere of Daily Life (NSDL)," created by MHLW, with modifications by the engaged public health researchers, in July 29, 2011. The details of the NSDL have been described previously. ${ }^{4-6}$ The target population of the current cluster RCT is individuals of the 19,424 aged $\geq 65$ years who did not have LTC certification ( $n=16,474$, Figure 1$)$.

We randomly selected 10 intervention regions from a total of 21 regions in Kameoka City (Cluster-RCT \#1). On February 13, 2012, we sent postal invitations to the people living in the intervention regions who had responded to the NSDL survey to attend a physical check-up. The check-up was conducted at a community center in each intervention region so that participants could access it easily and in familiar surroundings. The physical check-up was conducted during March and April, and an additional examination was conducted in September 2012. The purpose, procedures, and risks of the physical check-up were explained to each participant. All participants provided written informed consent before undergoing the check-up.

We allocated the 10 intervention regions randomly into home-based self-care with or without weekly class-styled sessions. The program in all the regions included a daily log (see Supplementary materials) maintenance by participants and monthly communications (to be described later) from the research staff by mail. Half of the regions (home-based intervention [HB] group, five regions) received only this program; the other half (class-styled intervention [CS] group, five regions) received the additional component of a weekly class-style session at the community center (Cluster-RCT \#2, Figures 2 and 3). After the physical check-up, we held the group meeting in each region to report the results of the check-up and to explain the CGIP for each region, so that the participants cannot select the program technically. Both the HB and CS interventions took place over 12 weeks, from May to August, or from September to December, according to class capacity and human resource availability. The baseline physical check-up examination was conducted in September for those who began the intervention in that month. The purpose, procedures, and risks of the intervention 


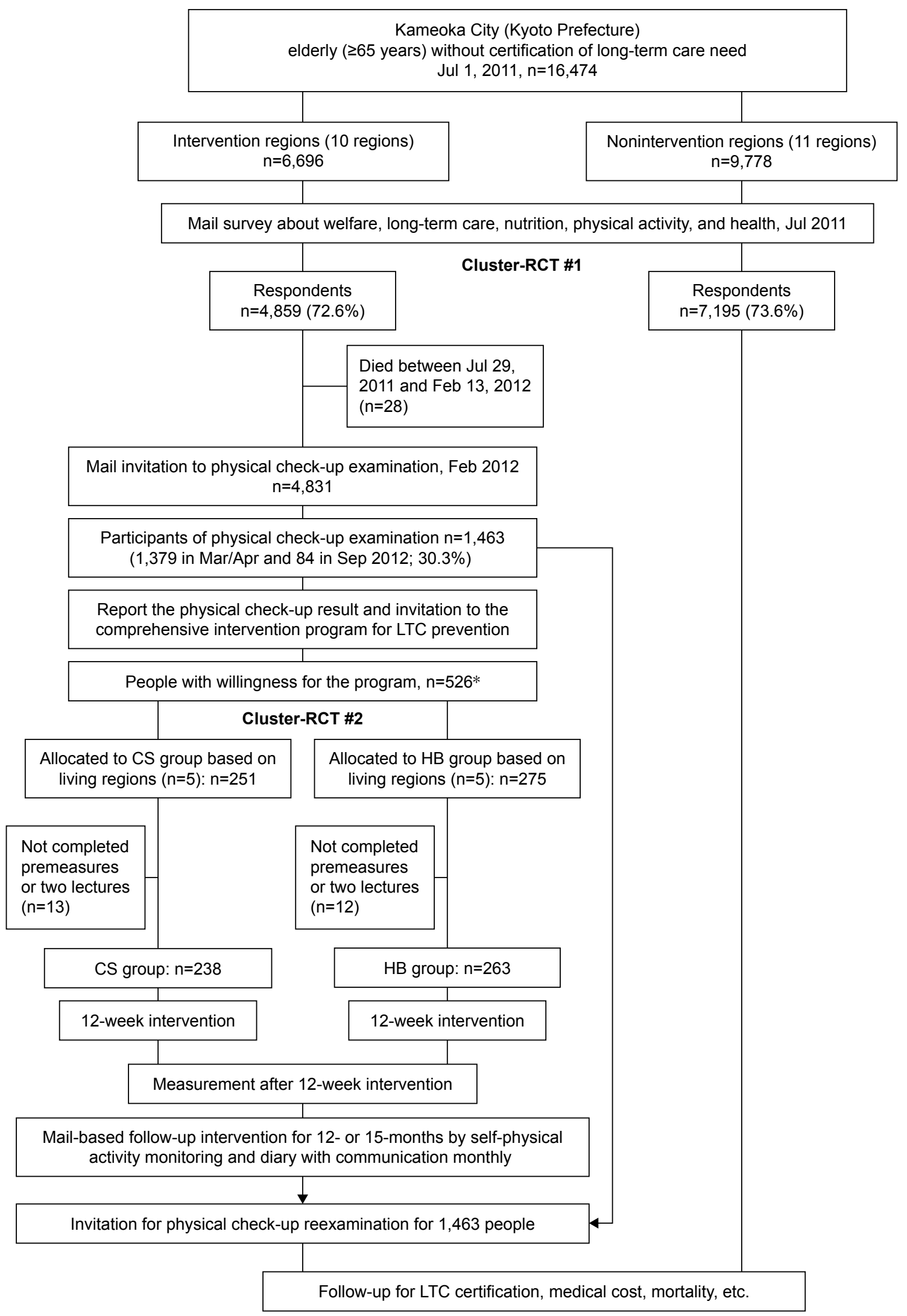

Figure I Flowchart of the current cluster-RCT Study.

Notes: *Two older adults received our intervention service despite not participating in the physical check-up examination at Mar/Apr and/or Sep 20I2. The two older adults were excluded from the analysis because of not completing premeasures.

Abbreviations: CS, class-styled; HB, home-based; LTC, long-term care; RCT, randomized controlled trial. 




Figure 2 Geographic features of Kameoka City, Kyoto Prefecture, Japan. Abbreviations: CS, class-styled; HB, home-based.

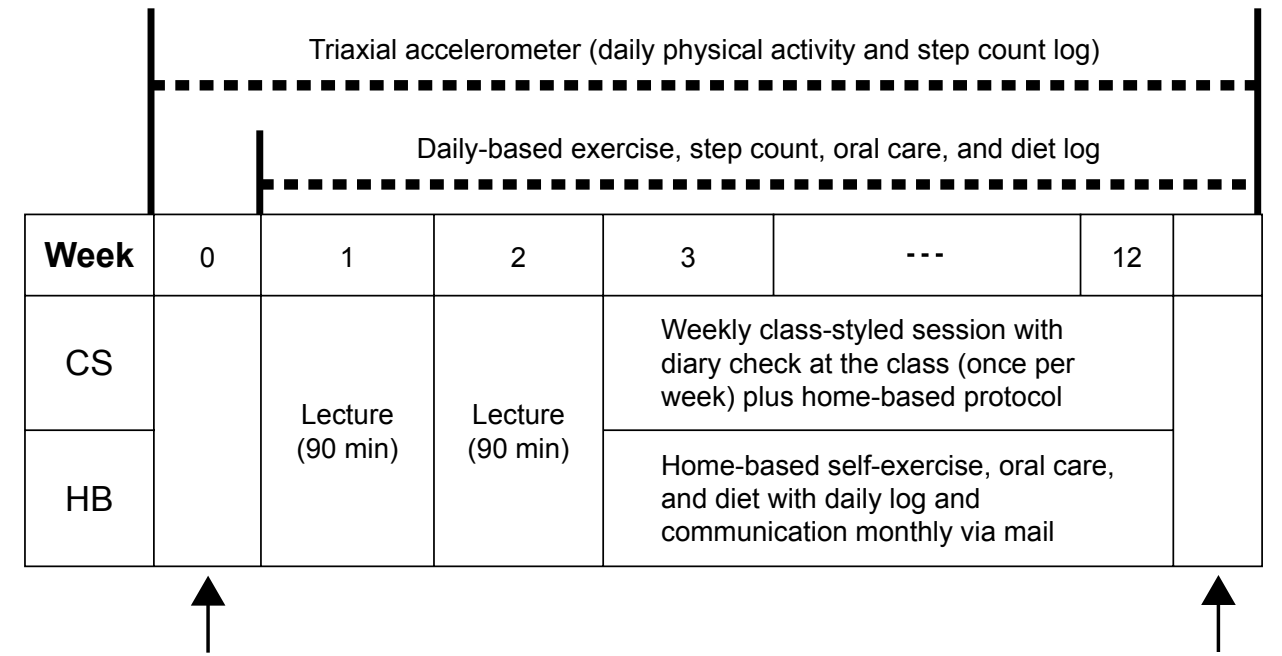

Baseline physical function test, physical activity measurement

Post physical function test, physical activity measurement

Figure 3 Scheme of HB or CS intervention.

Abbreviations: CS, class-styled; HB, home-based. 
were explained to each participant. All participants provided written informed consent before entering the CGIP. After the 12-week intervention, their physical function was reevaluated in order to examine the short-term effect of intervention.

Participants were instructed to keep a daily log of their step counts, exercise, diet, and oral care, and to post these to the research office. The research staff and volunteers returned comments to participants about their logs, and also provided a new month's supply of daily log sheets and monthly newsletter with geriatric health information. These monthly postal communications continued until the end of 2013.

The main purpose of this cluster RCT is to clarify the effect of the CGIP on LTC certification, LTC cost, survival time, health outcomes, and medical costs. The control group for this analysis will be selected from nonintervention regions (Cluster-RCT \#1; 11 regions, n=7,195; Figures 1 and 2). People in nonintervention regions were not invited to the physical check-up and CGIP. Another main purpose is to investigate the long-term effect of the CGIP on physical function. In this case, the control group is those who do not intend to participate in the CGIP in intervention regions (Cluster-RCT \#2; 10 regions; Figures 1 and 2). At the end of the follow-up period (October and November in 2013), we invited all participants in the physical check-up in 2012 for reexamination (Figure 2).

\section{Comprehensive geriatric intervention program}

The main intervention program was based on the participants' daily logs and included instructions on: 1) very low-load resistance training with suitable modification of previous program for use with our participants ${ }^{7}$ (Table 1);2) increasing daily physical activity and step count based on the ActiveGuide by MHLW; ${ }^{8-11} 3$ ) oral motor exercise and care; ${ }^{12-15}$ and 4) self-monitoring a well-balanced diet based on the Japanese Food Guide Spinning Top by MHLW. ${ }^{16-19}$ Details of the CGIP are provided in Supplementary materials.

\section{Outcomes}

\section{Physical activity}

The total number of step counts per day was evaluated using a triaxial accelerometer. ${ }^{20-22}$ Moderate-to-vigorous physical activity (MVPA), defined as $\geq 3$ metabolic equivalents (METs), was also obtained by the accelerometer. This measure has been validated and reported previously according to the Exercise and Physical Activity Guide for Health Promotion 2006 and 2013 established by MHLW. ${ }^{8-11}$ MVPA was obtained as "Exercise (Ex)" defined as METs h week ${ }^{-1} .^{8-11}$ The accelerometers were distributed 1 week before the intervention, and all participants were asked to wear them throughout the intervention period, exclusive of time spent in water. Mean number of daily steps in the week before the intervention, in the last week of the intervention, and at the end of the follow-up period were compared. When there were no pre-intervention data, the daily steps in the first and/or second week of the intervention were substituted. The first and the last days of the intervention period and the days with step counts $\leq 300$ were excluded from analysis. Participants who had at least four valid days were included in the analysis. These criteria followed a previous study. ${ }^{23}$ In accordance with the Exercise and Physical

Table I The low-load resistance exercise program

\begin{tabular}{|c|c|c|c|}
\hline Exercises & Exercise movement & Load & Volume \\
\hline \multicolumn{4}{|l|}{ Lower body } \\
\hline Stepping exercise & As quickly as possible & Ankle weight & $10 \mathrm{~s} \times 3-5$ sets \\
\hline \multirow[t]{2}{*}{ Single leg raise } & $\begin{array}{l}\text { Slow: } 3 \text {-s concentric, } 3 \text {-s eccentric, } \\
\text { and I-s isometric actions }\end{array}$ & Ankle weight & Slow: $6-10$ reps $\times 1-2$ sets \\
\hline & Quick: as quickly as possible & & Quick: 10 reps $\times 1$ set \\
\hline \multirow[t]{2}{*}{ Squat } & $\begin{array}{l}\text { Slow: } 3 \text {-s concentric, } 3 \text {-s eccentric, } \\
\text { and I-s isometric actions }\end{array}$ & Body weight & Slow: $6-10$ reps $\times 1-2$ sets \\
\hline & Quick: as quickly as possible & & Quick: 10 reps $\times 1$ set \\
\hline \multirow[t]{3}{*}{ Heel raise } & Slow: 2-s concentric and & Body weight & Slow: $10-15$ reps $\times 1-2$ sets \\
\hline & 2-s eccentric actions & & \\
\hline & Quick: as quickly as possible & & Quick: 15 reps $\times 1$ set \\
\hline \multicolumn{4}{|l|}{ Upper body } \\
\hline Arm curl & 4-s concentric and 4-s eccentric & Elastic band & $6-10$ reps $\times I-2$ sets \\
\hline Front raise & 4-s concentric and 4-s eccentric & Elastic band & $6-10$ reps $\times 1-2$ sets \\
\hline Side raise & 4-s concentric and 4-s eccentric & Elastic band & $6-10$ reps $\times I-2$ sets \\
\hline Shoulder external rotation & 4-s concentric and 4-s eccentric & Elastic band & $6-10$ reps $\times 1-2$ sets \\
\hline
\end{tabular}

Abbreviation: rep, repetitions. 
Activity Guide, ${ }^{8-11}$ we calculated the percentage of those with $>10 \mathrm{Ex} /$ week and $>23 \mathrm{Ex} /$ week.

\section{Physical function}

Physical function parameters were measured before and after the intervention, and at the end of the follow-up period. Main variables were the right front thigh muscle thickness, grip strength, maximum isometric right knee extension strength, usual and maximum walking velocity, the time from the timed up and go test, the five-time chair standing time, and vertical jump index (vertical jump height $\times$ body weight). Details of the measurements have been described previously. ${ }^{24-29}$

\section{Other secondary variables}

We also assessed with a Health and Nutrition survey with the following information after 12- or 15-months follow-up: 1) demographics (living arrangements, need for LTC, social network, and social support); 2) activities of daily living (International Physical Activity Questionnaire Environment module); ${ }^{30,31} 3$ ) general and mental health (height, weight, self-rated health, geriatric depression scale [GDS-15], ${ }^{32}$ and [WHO-5]);33 4) lifestyle profiles (smoking status, alcohol status, and Pittsburgh Sleep Quality Index); ${ }^{34} 5$ ) oral health (General Oral Health Assessment Index); ${ }^{35,36}$ 6) nutrition status (risk evaluation for eating and nutrition [SCREEN II] $]^{37}$ and Food Frequency Questionnaire). ${ }^{38-41}$ Furthermore, we measured total daily energy expenditure before and after intervention using the doubly labeled water methods ${ }^{42}$ for small subsamples.

\section{LTC data and mortality}

The collection of follow-up data will be conducted periodically for an indefinite period. We will follow five types of outcomes: LTC certification, LTC cost, survival time, health outcomes, and medical costs. The follow-up survey will be completed 2 years after the baseline. We are conducting this prospective cohort study with the cooperation of the Kameoka City municipal government, and using the residential registry of Kameoka City to follow the cohort participants for mortality and relocation out of the city.

\section{Results}

In cluster RCT \#1, there were 6,696 elderly in the 10 intervention regions and 9,778 elderly in the 11 nonintervention regions who did not have LTC certification. Of these, 4,859 $(72.6 \%)$ and $7,195(73.6 \%)$ responded to the NSDL survey, respectively, and there was no difference in response rates. A total of 4,831 people in the intervention regions were invited for the physical check-up, and 1,463 (30.3\%) participated. After receiving their check-up results and an explanation of the intervention program, 526 people expressed willingness to participate. Of these, 275 were allocated to the $\mathrm{HB}$ group and 251 to the CS group according to their region of residence. Once allocated, 263 people in the $\mathrm{HB}$ group and 238 in the CS group completed premeasures and two lectures (see Supplementary materials) and began the 12-week intervention.

Table 2 shows the baseline characteristics of the participants in each group. A significant interaction was observed in daily step counts by two-way analysis of variance (group $\times$ time).

Table 2 Baseline physical characteristics and daily step count in the intervention participants

\begin{tabular}{|c|c|c|c|}
\hline \multirow[t]{2}{*}{ Variables } & \multirow{2}{*}{$\begin{array}{l}\text { CS }(n=238) \\
\text { Mean } \pm \text { SD }\end{array}$} & \multirow{2}{*}{$\begin{array}{l}\text { HB }(n=263) \\
\text { Mean } \pm \text { SD }\end{array}$} & \multirow[t]{2}{*}{$P$-value } \\
\hline & & & \\
\hline Age (years) & $73.9 \pm 5.4$ & $74.1 \pm 5.6$ & 0.767 \\
\hline Number of women (\%) & $138(58.0)$ & $156(59.3)$ & 0.762 \\
\hline Height $(\mathrm{cm})$ & $156.2 \pm 8.6$ & $156.4 \pm 8.3$ & 0.733 \\
\hline Weight (kg) & $55.7 \pm 9.9$ & $55.9 \pm 9.4$ & 0.862 \\
\hline Body mass index $\left(\mathrm{kg} / \mathrm{m}^{2}\right)$ & $22.8 \pm 3.2$ & $22.8 \pm 3.0$ & 0.954 \\
\hline Daily step counts (steps/day) & $5,005.6 \pm 2,782.2$ & $4,892.4 \pm 2,898.8$ & 0.662 \\
\hline Front thigh muscle thickness $(\mathrm{mm})$ & $41.6 \pm 6.5$ & $42.5 \pm 6.5$ & 0.138 \\
\hline Grip strength (kg) & $27.6 \pm 8.2$ & $28.1 \pm 8.1$ & 0.565 \\
\hline Maximum isometric knee & $28.0 \pm 11.0$ & $29.6 \pm 11.2$ & 0.101 \\
\hline \multicolumn{4}{|l|}{ extension strength (kg) } \\
\hline Usual walking velocity $(\mathrm{m} / \mathrm{s})$ & $1.35 \pm 0.24$ & $1.40 \pm 0.25$ & 0.028 \\
\hline Maximum walking velocity $(\mathrm{m} / \mathrm{s})$ & $1.82 \pm 0.32$ & $1.85 \pm 0.34$ & 0.259 \\
\hline Time of timed up and go test (s) & $7.53 \pm 2.00$ & $7.04 \pm 1.71$ & 0.004 \\
\hline Five-time chair standing time (s) & $8.31 \pm 2.62$ & $8.26 \pm 2.57$ & 0.843 \\
\hline Vertical jump index (m×kg) & $12.1 \pm 5.2$ & $12.3 \pm 5.4$ & 0.713 \\
\hline
\end{tabular}

Abbreviations: CS, class-styled; HB, home-based. 
Mean number of daily steps was significantly increased from the baseline in both groups. The increase in daily steps in the CS group was significantly greater than that in the HB group (CS, 1,318.3 steps/day, HB, 701.1 steps/day; $P=0.006$ ).

\section{Discussion}

We were able to launch the cluster RCT with a good response rate in each phase.

In the current intervention, participants increased their step count $\sim 1,000$ per day (+10 $\mathrm{min}$ ) on average from the baseline and conducted daily-based self-care with daily log at the program. Miyachi et al conducted a meta-analysis and reported that an increment of 10 min of MVPA per day can result in a $3.2 \%$ reduction of the average relative risk of noncommunicable diseases, dementia, joint musculoskeletal impairment, and mortality. Therefore, we expect that this intervention can contribute to the health promotion of the participants in both groups. ${ }^{11}$ However, we have to remain neutral scientifically for the effect of current intervention before the data are analyzed and published.

This intervention program contains the factors that promote muscle hypertrophy. A previous study reported the muscle hypertrophic effect of a 12 -week intervention with a slightly weighted (200 600 g) shoe, ${ }^{43}$ so we provided a single set of $500 \mathrm{~g}$ ankle weights to all participants in the present study. Previous studies reported that low-load (30\%-60\%, one repetition maximum) resistance training with slow movement (3-s concentric, 3-s eccentric and 1-s isometric actions) and sustained contractile force caused significant increases in muscle size and strength in untrained young men and robust elderly participants. ${ }^{44-46}$ The slow movement protocol was applied to resistance training program of the intervention. Although low-load resistance training with slow movement and sustained contractile force is effective for increasing muscle size, this protocol has an unfavorable effect on dynamic sports movements. ${ }^{47}$ Thus, this type of resistance training might be ineffective for improving motor functions in activities of daily life, such as walking and climbing stairs, for older individuals. In the present study, a stepping exercise that required stepping as quickly as possible for $10 \mathrm{~s}$ was included to improve physical function. Also, all participants were instructed to perform a single set (10-15 repetitions) of the three exercises (single leg raise, squat, and cuff raise) as quickly as possible after fifth week of the 12-week intervention period in order to improve total physical function. In addition to MVPA and resistance exercise, oral care and nutritional factor were included in the current intervention program. Approaches to oral function and nutrition may additionally contribute to muscle hypertrophy and improvement of physical function.

As MHLW recommended, our intervention program is comprehensive. It consisted of increasing physical activity, practical resistance training, oral care, and nutritional lecture. Each factor is important for health promotion of older population. We believe that our CGIP is feasible for older people. This study is an attempt to verify whether the CGIP can be used as a large population approach to communitydwelling elderly for health promotion. On the other hand and also the limitation of this study, we cannot evaluate the contribution of individual factor included in the program, because this study was designed to investigate the effect of the comprehensive program.

\section{Conclusion}

We successfully launched a cluster RCT with good response rates in each phase. This is the first report about our CGIP in Kyoto-Kameoka Study. Participants increased their step count by $\sim 1,000$ per day $(+10 \mathrm{~min})$ in the program. This RCT will provide valuable information and evidence about a comprehensive, community-based geriatric intervention program. Supplementary materials will be freely available for developing community-based CGIP.

\section{Acknowledgments}

The authors thank all the participants in this study as well as Kameoka City, The Promotion Organization of Integrated Community Care System of Kyoto Prefecture, Kyoto Dental Hygienists' Association, Kyoto Dietetic Association, Kameoka City municipal government, and all staff of the Kameoka-Study Group. We especially thank Dr Motoko Miyake, Kyoto Gakuen University; Prof Taeko Masumoto and Prof Yasuko Okayama, Doshisha Women's College of Liberal Arts; Ms Miho Nishimura and Mr Hajime Tamiya, Kyoto Prefectural University of Medicine; Prof Tomoki Nakaya, Ritsumeikan University; Dr Minoru Yamada, Tsukuba University; Prof Hidenori Arai, National Center for Geriatrics and Gerontology; Dr Mitsuyoshi Yoshida, Hiroshima University; Prof Takeshi Kikutani, Nippon Dental University; Dr Hinako Nanri, National Institutes of Biomedical Innovation, Health and Nutrition; Dr Hiroyuki Sagayama, Japan Society for the Promotion of Science; Dr Date Heiwa, Shiga University; Mr Masahiro Fukuda, Ms Kazuko Ohkura, Ms Haruka Nishioka, and Ms Sairi Watanabe for their dedicated efforts. This work was supported by MEXT KAKENHI research grants from JSPS (Grant Numbers 24240091 [to MK], 25750360 and 
15K16531 [to YW], 23-333 and 15H05363 [to YY]) and by a grant from the Promotion Organization of Integrated Community Care System of Kyoto Prefecture and a grant from the Kameoka City municipal government.

\section{Disclosure}

The authors report no conflicts of interest in this work.

\section{References}

1. Campbell JC, Ikegami N. Long-term care insurance comes to Japan. Health Aff (Millwood). 2000;19(3):26-39.

2. Health and Welfare Bureau for the Elderly, Ministry of Health, Labour and Welfare. Long-Term Care Insurance System of Japan. 2016. Available from: http://www.mhlw.go.jp/english/policy/care-welfare/ care-welfare-elderly/d1/ltcisj_e.pdf. Accessed April 25, 2018.

3. Ministry of Health, Labour and Welfare. Manual for Prevention of Long-Term Care in Elderly (Revised). 2012. Japanese.

4. Yamada Y, Nanri H, Watanabe Y, et al. Prevalence of frailty assessed by Fried and Kihon checklist indices in a prospective cohort study: design and demographics of the Kyoto-Kameoka longitudinal study. J Am Med Dir Assoc. 2017;18:733.e7-733.e15.

5. Yamagata E, Kimura M, Miyake M, et al. Relationship between homebound status and physical fitness in the community-dwelling elderly population. [Nihon koshu eisei zasshi] Jpn J Public Health. 2014;61(11):671-678.

6. Masumoto T, Yamada Y, Yamada M, et al. Fall risk factors and sex differences among community-dwelling elderly individuals in Japan. A Kameoka study. [Nihon koshu eisei zasshi] Jpn J Public Health. 2015;62(8):390-401.

7. Watanabe Y, Tanimoto M, Oba N, Sanada K, Miyachi M, Ishii N. Effect of resistance training using bodyweight in the elderly: comparison of resistance exercise movement between slow and normal speed movement. Geriatr Gerontol Int. 2015;15(12):1270-1277.

8. Ministry of Health, Labour and Welfare. Exercise and Physical Activity Reference for Health Promotion 2006, Report in Japanese, 2006.

9. Ministry of Health, Labour and Welfare. Physical Activity References for Health Promotion 2013, Report in Japanese, 2013.

10. Murakami H, Tripette J, Kawakami R, Miyachi M. "Add 10 min for your health": the new Japanese recommendation for physical activity based on dose-response analysis. J Am Coll Cardiol. 2015;65(11):1153-1154.

11. Miyachi M, Tripette J, Kawakami R, Murakami H. “+10 min of physical activity per day": Japan is looking for efficient but feasible recommendations for its population. J Nutr Sci Vitaminol. 2015;61(Suppl):S7-S9.

12. Watanabe $\mathrm{Y}$, Hirano $\mathrm{H}$, Arai $\mathrm{H}$, et al. Relationship between frailty and oral function in community-dwelling elderly adults. $J$ Am Geriatr Soc. 2017;65(1):66-76.

13. Tamura F, Kikutani T, Tohara T, Yoshida M, Yaegaki K. Tongue thickness relates to nutritional status in the elderly. Dysphagia. 2012;27(4): $556-561$.

14. Sagawa K, Kikutani T, Tamura F, Yoshida M. Factors related to skeletal muscle mass in the frail elderly. Odontology. 2017;105(1):91-95.

15. Kikutani T, Yoneyama T, Nishiwaki K, Tamura F, Yoshida M, Sasaki H. Effect of oral care on cognitive function in patients with dementia. Geriatr Gerontol Int. 2010;10(4):327-328.

16. Ministry of Health, Labour and Welfare and Ministry of Agriculture, Forestry and Fisheries. Japanese Food Guide Spinning Top. 2005. Available from: http://www.maff.go.jp/j/balance_guide/b_use/pdf/ eng_reiari.pdf. Accessed April 25, 2018.

17. Yoshiike N, Hayashi F, Takemi Y, Mizoguchi K, Seino F. A new food guide in Japan: the Japanese food guide Spinning Top. Nutr Rev. 2007; 65(4):149-154.

18. Oba S, Nagata C, Nakamura K, et al. Diet based on the Japanese Food Guide Spinning Top and subsequent mortality among men and women in a general Japanese population. J Am Diet Assoc. 2009;109(9): $1540-1547$.
19. Kurotani K, Akter S, Kashino I, et al. Quality of diet and mortality among Japanese men and women: Japan Public Health Center based prospective study. BMJ. 2016;352:i1209.

20. Yamada Y, Yokoyama K, Noriyasu R, et al. Light-intensity activities are important for estimating physical activity energy expenditure using uniaxial and triaxial accelerometers. Eur J Appl Physiol. 2009; 105(1):141-152.

21. Yamada Y, Yokoyama K, Noriyasu R, et al. Erratum to: Calculation of total energy expenditure in publications on physical activity energy by Yamada et al. in 2009 and 2013. Eur J Appl Physiol. 2016;116(6):1279-1280.

22. Murakami H, Kawakami R, Nakae S, et al. Accuracy of wearable devices for estimating total energy expenditure: comparison with metabolic chamber and doubly labeled water method. JAMA Intern Med. 2016;176(5):702-703.

23. Ikenaga M, Yamada Y, Takeda N, et al. Dynapenia, gait speed and daily physical activity measured using triaxial accelerometer in older Japanese men. Jpn J Phys Fitness Sports Med. 2014;3:147-154.

24. Kimura M, Mizuta C, Yamada Y, Okayama Y, Nakamura E. Constructing an index of physical fitness age for Japanese elderly based on 7-year longitudinal data: sex differences in estimated physical fitness age. Age (Dordr). 2012;34(1):203-214.

25. Yamada Y, Yoshida T, Yokoyama K, et al. The extracellular to intracellular water ratio in upper legs is negatively associated with skeletal muscle strength and gait speed in older people. J Gerontol A Biol Sci Med Sci. 2017;72(3):293-298.

26. Yamada Y, Watanabe Y, Ikenaga M, et al. Comparison of single- or multifrequency bioelectrical impedance analysis and spectroscopy for assessment of appendicular skeletal muscle in the elderly. $J$ Appl Physiol. 2013;115(6):812-818.

27. Yamada Y, Schoeller DA, Nakamura E, Morimoto T, Kimura M, Oda S. Extracellular water may mask actual muscle atrophy during aging. J Gerontol A Biol Sci Med Sci. 2010;65A(5):510-516.

28. Watanabe Y, Yamada Y, Fukumoto Y, et al. Echo intensity obtained from ultrasonography images reflecting muscle strength in elderly men. Clin Interv Aging. 2013;8:993-998.

29. Yoshida T, Kimura M, Watanabe Y, et al. Differences in frailty prevalence between evaluating methods in 1,379 community-dwelling older adults-Kyoto-Kameoka Study. Jpn J Health Promot. 2017;19(1):9-18.

30. Craig CL, Marshall AL, Sjostrom M, et al. International physical activity questionnaire: 12-country reliability and validity. Med Sci Sports Exerc. 2003;35(8):1381-1395.

31. Rosenberg DE, Bull FC, Marshall AL, Sallis JF, Bauman AE. Assessment of sedentary behavior with the International Physical Activity Questionnaire. J Phys Act Health. 2008;5(Suppl 1):S30-S44.

32. Schreiner AS, Hayakawa H, Morimoto T, Kakuma T. Screening for late life depression: cut-off scores for the Geriatric Depression scale and the Cornell Scale for Depression in Dementia among Japanese subjects. Int J Geriatr Psychiatry. 2003;18(6):498-505.

33. Awata S, Bech P, Koizumi Y, et al. Validity and utility of the Japanese version of the WHO-Five Well-Being Index in the context of detecting suicidal ideation in elderly community residents. Int Psychogeriatr. 2007;19(1):77-88.

34. Buysse DJ, Reynolds CF 3rd, Monk TH, Berman SR, Kupfer DJ. The Pittsburgh Sleep Quality Index: a new instrument for psychiatric practice and research. Psychiatry Res. 1989;28(2):193-213.

35. Atchison KA, Dolan TA. Development of the geriatric oral health assessment index. J Dent Educ. 1990;54(11):680-687.

36. Naito M, Yuasa H, Nomura Y, Nakayama T, Hamajima N, Hanada N. Oral health status and health-related quality of life: a systematic review. J Oral Sci. 2006;48(1):1-7.

37. Keller HH, Goy R, Kane SL. Validity and reliability of SCREEN II (Seniors in the community: risk evaluation for eating and nutrition, Version II). Eur J Clin Nutr. 2005;59(10):1149-1157.

38. Goto C, Tokudome Y, Imaeda N, et al. Validation study of fatty acid consumption assessed with a short food frequency questionnaire against plasma concentration in middle-aged Japanese people. Scand J Nutr. 2006;50(2):77-82. 
39. Imaeda N, Goto C, Tokudome Y, Hirose K, Tajima K, Tokudome S. Reproducibility of a short food frequency questionnaire for Japanese general population. J Epidemiol. 2007;17(3):100-107.

40. Tokudome S, Goto C, Imaeda N, Tokudome Y, Ikeda M, Maki S. Development of a data-based short food frequency questionnaire for assessing nutrient intake by middle-aged Japanese. Asian Pac J Cancer Prev. 2004;5(1):40-43.

41. Tokudome Y, Goto C, Imaeda N, et al. Relative validity of a short food frequency questionnaire for assessing nutrient intake versus three-day weighed diet records in middle-aged Japanese. J Epidemiol. 2005; 15(4):135-145.

42. Schoeller DA, Ravussin E, Schutz Y, Acheson KJ, Baertschi P, Jequier E. Energy expenditure by doubly labeled water: validation in humans and proposed calculation. Am J Physiol Regul Integr Comp Physiol. 1986;250(5):R823-R830.

43. Ikenaga M, Yamada Y, Mihara R, et al. Effects of slightly-weighted shoe intervention on lower limb muscle mass and gait patterns in the elderly. Jpn J Phys Fitness Sports Med. 2012;61(5):469-477.
44. Watanabe Y, Tanimoto M, Ohgane A, Sanada K, Miyachi M, Ishii N. Increased muscle size and strength from slow-movement, low-intensity resistance exercise and tonic force generation. J Aging Phys Act. 2013; 21(1):71-84.

45. Watanabe Y, Madarame H, Ogasawara R, Nakazato K, Ishii N. Effect of very low-intensity resistance training with slow movement on muscle size and strength in healthy older adults. Clin Physiol Funct Imaging. 2014;34(6):463-470.

46. Tanimoto M, Ishii N. Effects of low-intensity resistance exercise with slow movement and tonic force generation on muscular function in young men. J Appl Physiol (1985). 2006;100(4):1150-1157.

47. Tanimoto M, Arakawa H, Sanada K, Miyachi M, Ishii N. Changes in muscle activation and force generation patterns during cycling movements because of low-intensity squat training with slow movement and tonic force generation. J Strength Cond Res. 2009;23(8):2367-2376. 


\section{Supplementary materials Details on the comprehensive geriatric intervention program}

Exercise and physical activity

A triaxial accelerometer/pedometer (EW-NK52; Panasonic Electric Works, Tokyo, Japan), a single set of $500 \mathrm{~g}$ ankle weights (STW140; Sinwa Enterprise, Osaka, Japan), and an elastic band (Thera-Band, Hygenic Co., Akron, OH, USA) were provided free of charge as exercise materials to all participants. The total cost of these materials was about $\$ 30$ per person. Three different resistance levels of elastic band (Yellow $<\operatorname{Red}<$ Green) were offered, and the participants selected one at their discretion.

The low-load resistance training program consisted of eight exercises (stepping, squats, single leg raises, heel raises, arm curls, front raises, side raises, and shoulder external rotations). Details of the exercises are shown in Table 1. All exercises for the lower limbs were performed using bodyweight as the load. An ankle weight was used as an additional load for stepping exercises and single leg raises. All exercises for the upper limbs were performed using an elastic band as the load. A slow movement method ${ }^{1,2}$ was employed, with muscle contractions held for 7-8 s per repetition, for all exercises except stepping. After 5 weeks, a single set (10-15 reps) of three exercises (single leg raises without ankle weight, squats, and heel raises), which were performed as quickly as possible, were added to the resistance training program.

The daily step count was measured before the intervention, and the participants were instructed to increase their number of daily steps progressively. We recommended that steps be increased by up to 2,500 per day from the baseline, but at an individually comfortable rate of increasing steps. We instructed participants who already had daily step counts of 10,000 or more to maintain their physical activity. We also mentioned if participants could and wanted to impose an additional load in daily life, walking while wearing ankle weights was a good exercise. The ActiveGuide by MHLW recommends " +10 " that is "add 10 min of moderate-tovigorous physical activity (MVPA) per day" (Figure S1). ${ }^{3-5}$ In addition, the ActiveGuide recommends 40 min of any level of physical activity, including low-intensity physical activity, for the geriatric population aged $\geq 65$ years. The " +10 " equates to nearly 1,000 steps and the triaxial accelerometer can measure "Exercise (Ex)" (metabolic equivalents $[\mathrm{METs}] \times \mathrm{h}$ ) and total energy expenditure including low and moderate-to-vigorous levels.

\section{Oral function}

At first week of the intervention period, the participants took part in a lecture by a professional dental hygienist, and then were instructed to perform 5 min of oral motor exercise and oral care using a toothbrush. The oral motor exercise program was supervised by the Kyoto Dental Hygienists' Association. Figure $\mathrm{S} 2$ shows one of the leaflets from the oral motor exercise that was distributed to all participants.

\section{Nutrition and diet}

In Japan, MHLW and the Ministry of Agriculture, Forestry and Fisheries jointly developed the Japanese Food Guide Spinning Top in 2005 (Figure S3). ${ }^{6-9}$ This guide attempts to illustrate the balance and quantity of food in the recommended daily Japanese diet. The food guide prescribes the daily base of balanced intake of grains, vegetables, fish and meat, milk, and fruits. At second week of the intervention period, the participants took part in a lecture from a registered dietitian. To improve their nutritional status and physical function, and to prevent sarcopenia, frailty, and long-term care certification, we recommended grain dishes, vegetable dishes, and fish or meat dishes three times per day (breakfast, lunch, and dinner) and milk or milk products and fruits at least one time per day. The participants were instructed to check their diet habit on a daily log. We provided commercially available brochures and leaflets of educational material on nutrition and diet based on the Japanese Food Guide recommendations.

\section{Weekly class-style program}

The CS group attended 90 min weekly class-style sessions at a community center in each intervention region in addition to the previously mentioned home-based self-care. The weekly class-style session contained three to seven lowimpact dance exercises (maximum of $35 \mathrm{~min}$; 3-5 min for each exercise), oral motor exercise (5 min), and resistance exercise ( $40 \mathrm{~min})$. The HB group did not participate in any class-style sessions. Optional lectures and counseling were held in 5th to 6th weeks of the 12-week intervention period for the HB group to answer any questions they had. Otherwise, the participants in the HB group carried out the intervention program independently at home.

\section{Physical activity, exercise, oral care, and diet log}

The daily self-care $\log$ was provided to all participants (Figure S4). All participants were instructed to record their daily step counts, the resistance exercise, and oral motor 
+10 min of activity every day. anvwhore, anytime:

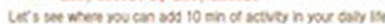
18 - 64 years old 65 years oc eldere

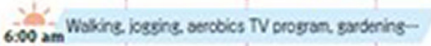

max comentent

Toke a bresk nak,

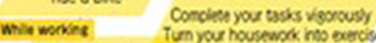



use the stars chose the batrocon

Dorne inatorax

Darme vorteresk

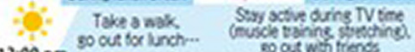

$12: 00$ pm was wores



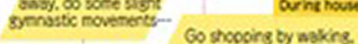

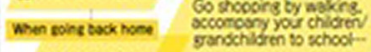

heresseyor

use the sars

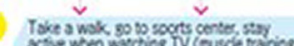

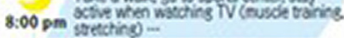

For your safety

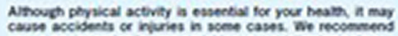

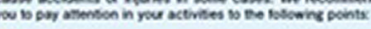

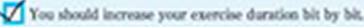

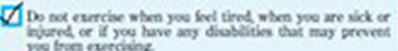

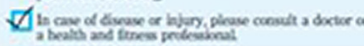

\section{Let's start with +10}

To have a longer healthy life, be active for an additional $10 \mathrm{~min}$ every day.
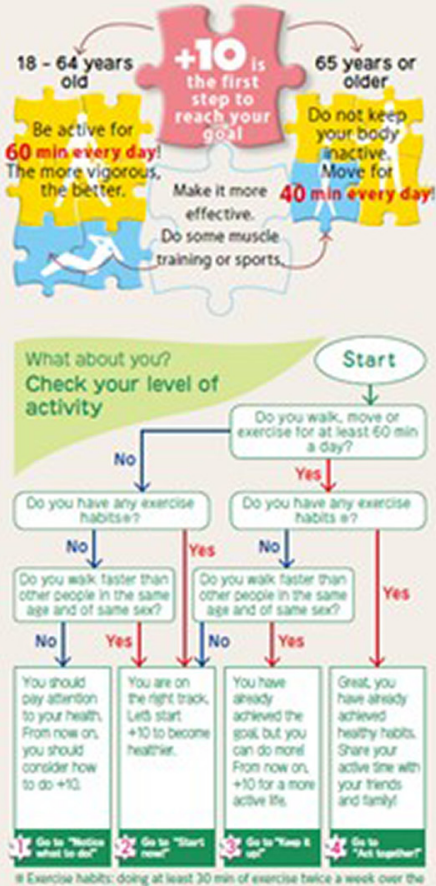

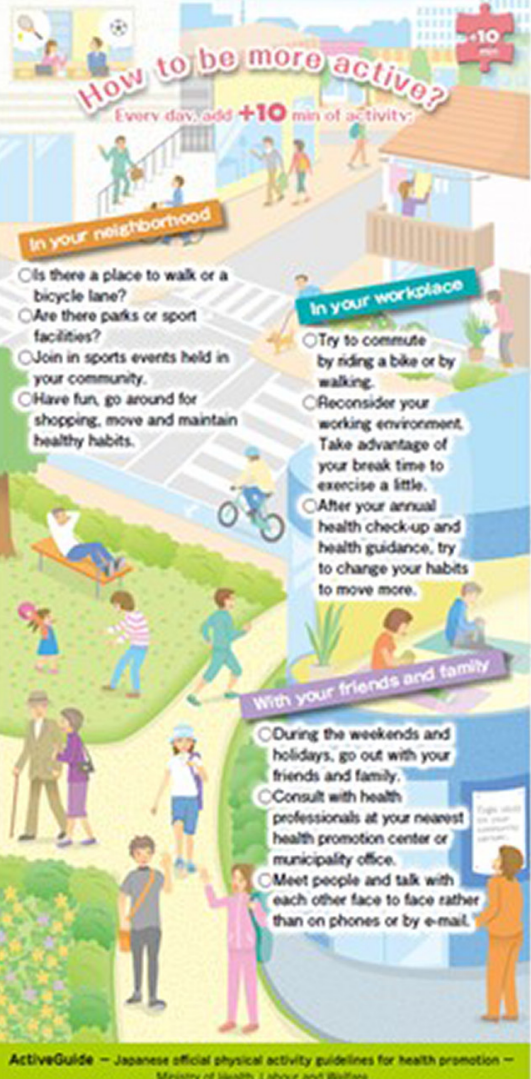

ActiveGuide

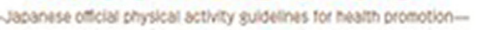

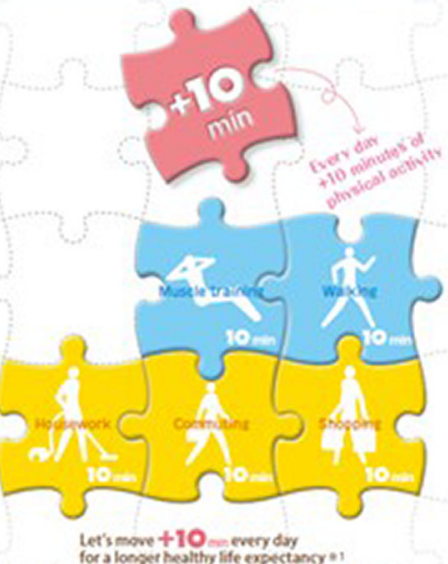

Be axtive and enioy a hea thy life. Physical activity lowers the risks of Giabests. Neart Giseast. stroke. cancer. why den ty you try to novet for an adstionst 10 min every $\sin$ ?

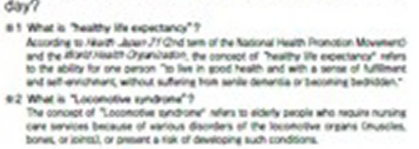

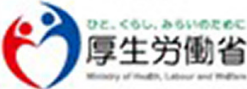

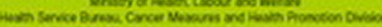

Your first steps for a healthy life!

13 Notice what to do!

Every dry you have many chances to become axtive in your dally life. Find when and where you can move for $10 \mathrm{~min}$ moce.

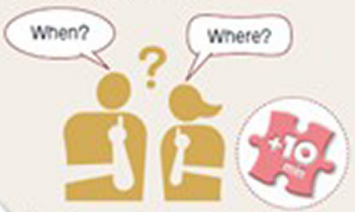

22 Start now!

The ferst step to get healthy is to move a little longer and more vigorously than what you do now. Start by adding $10 \mathrm{~min}$ of activity every day.

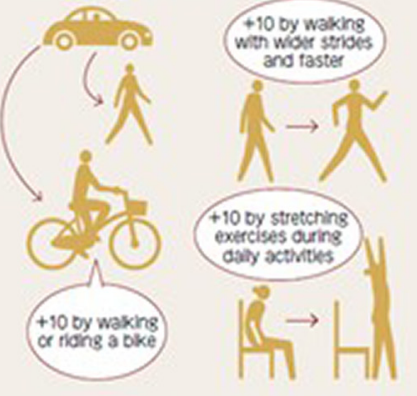

23 Keep it up!

Your goal is to move for 60 min every day (40 min for the eiderty Get fit

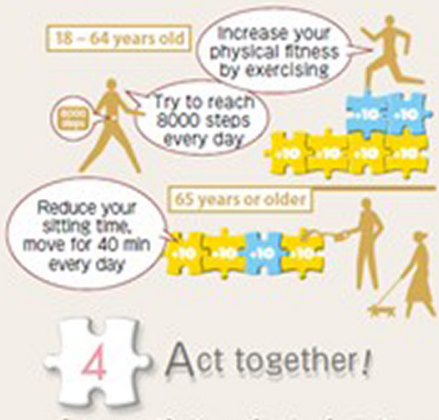

Share your +10 with your friends or family. it is moee fun to share your activities with someone else.



Figure SI ActiveGuide “+ 10 min" by Ministry of Health, Labour and Welfare.

Note: Reproduced from Ministry of Health, Labour and Welfare. Physical Activity References for Health Promotion 2013. Available from: http://www.nibiohn.go.jp/eiken/ info/pdf/active2013-e.pdf. 5 
exercise status (did or did not perform), and nutrition status as part of the log. In the CS group, we wrote comments to the logs at the weekly class-style sessions. We also collected the logs and checked them each month. In the HB group, the participants sent their logs to our laboratory each month via postal mail, and the logs were returned after we had checked them and written comments in response to the entries. We continued sending and collecting the logs, and replying with comments until the end of 2013 (for 12- or 15-months), after the 12-week intervention program had concluded.

\section{Oral motor exercise (oral-related preventive healthcare)}

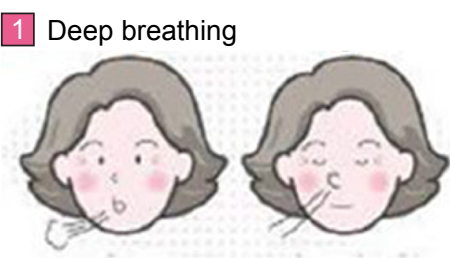

3 Shoulder exercise

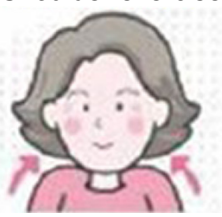

5 Cheeks exercise

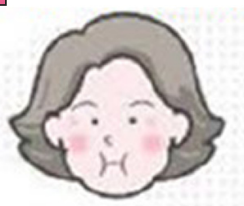

7 Tongue exercise

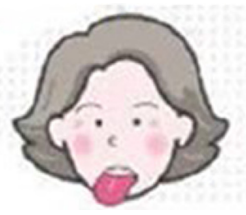

Cough exercise

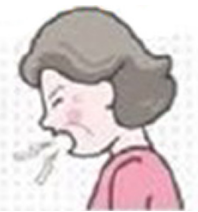

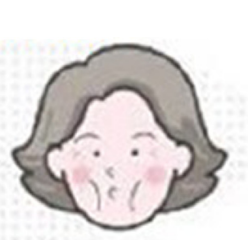
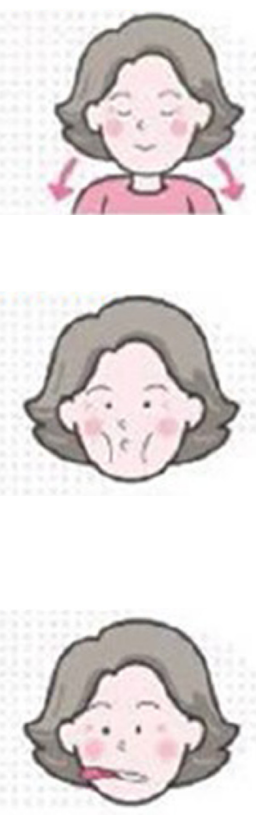

2 Rolling the neck


4 Jaw exercise
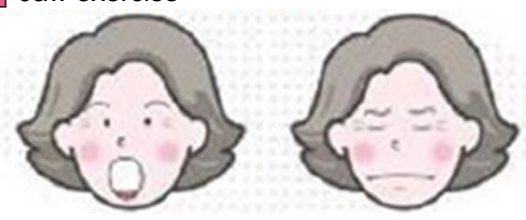

6 Lips exercise

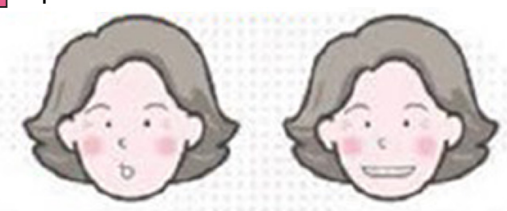

8 Salivary gland massaging
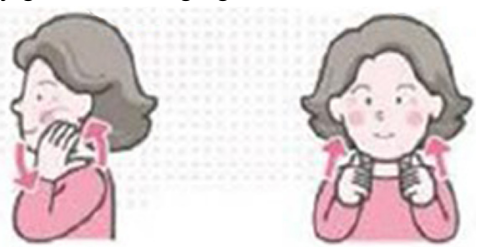

1 Vocal exercise

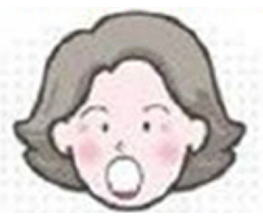

Figure S2 Oral motor exercise by Kyoto Dental Hygienists' Association. 


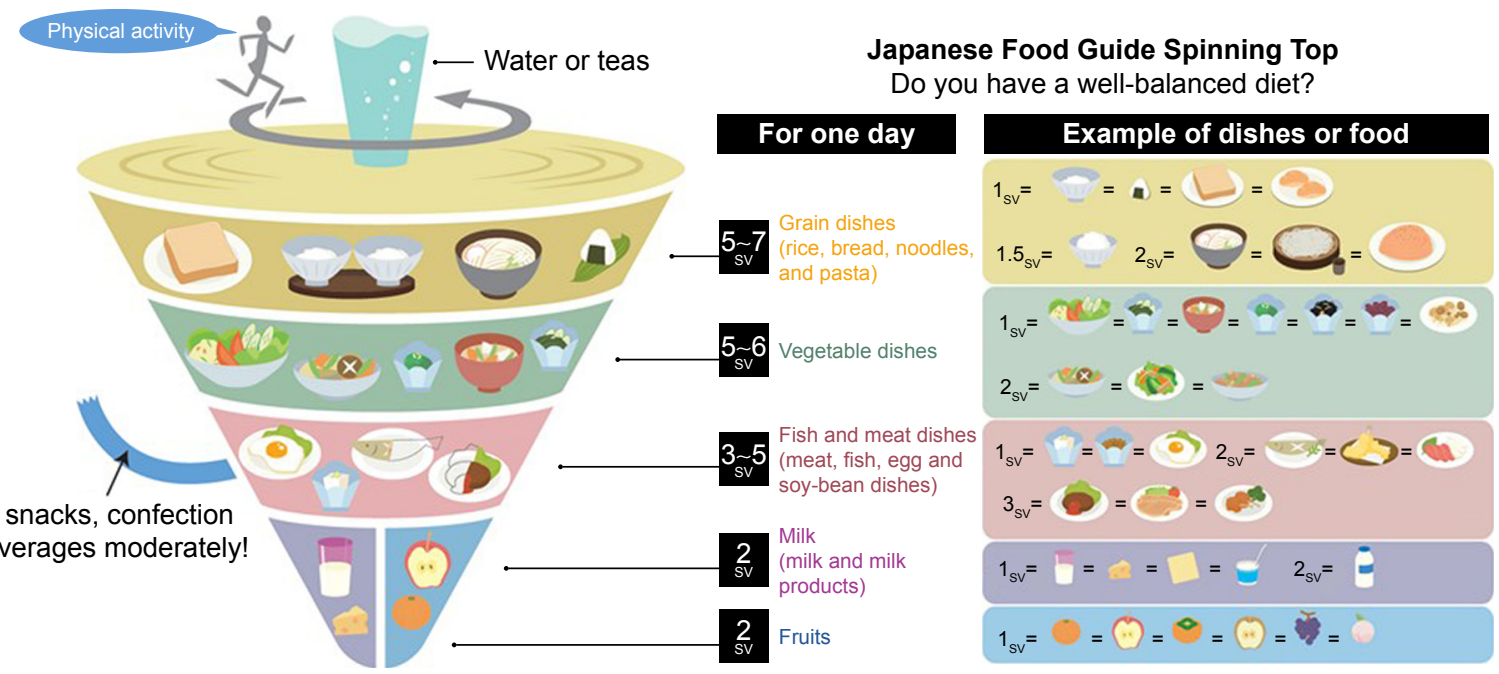

Figure S3 Japanese Food Guide Spinning Top (SV, "serving", simply countable number describing estimated amount of each dish or food served to one person determined by MHLW and Ministry of Agriculture, Forestry and Fisheries [MAFF]).

Notes: SV is an abbreviation of "serving", which is a simply countable number describing the approximated amount of each dish or food served to one person. Decided by Ministry of Health, Labour and Welfare and Ministry of Agriculture, Forestry and Fisheries. Reproduced from Ministry of Health, Labour and Welfare and Ministry of Agriculture, Forestry and Fisheries. Japanese Food Guide Spinning Top. 2005. Available from: http://www.maff.go.jp/j/balance_guide/b_use/pdf/eng_reiari.pdf.6 


\begin{tabular}{|c|c|c|c|c|c|c|c|c|c|c|c|}
\hline & \multicolumn{4}{|c|}{ Exercise } & \multicolumn{2}{|c|}{ Oral function } & \multicolumn{5}{|c|}{ Nutrition } \\
\hline & 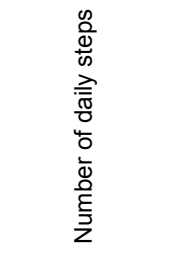 & 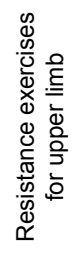 & 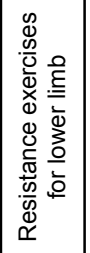 & 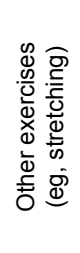 & 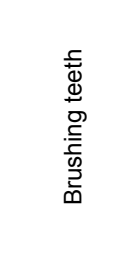 & 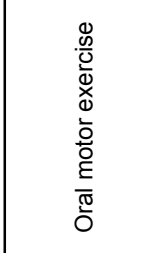 & 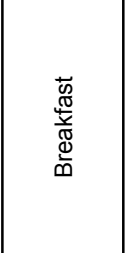 & $\begin{array}{l}\frac{1}{0} \\
\stackrel{5}{\Xi}\end{array}$ & 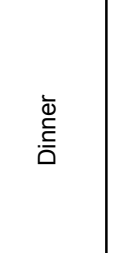 & $\begin{array}{l}\bar{\Phi} \\
\text { 志 }\end{array}$ & $\begin{array}{l}0 \\
\frac{0}{0} \\
\frac{0}{0} \\
\frac{\pi}{0} \\
0\end{array}$ \\
\hline & $\begin{array}{l}\text { Please write } \\
\text { before going } \\
\text { bed. }\end{array}$ & \multicolumn{3}{|c|}{$\begin{array}{l}\text { O: Did } \\
\mathbf{x} \text { : Did not }\end{array}$} & $\begin{array}{l}\text { 3: } 3 \text { times } \\
\text { over } \\
\text { 2: twice } \\
\text { 1: Once } \\
\text { 0: Did not }\end{array}$ & $\begin{array}{l}\text { O: Did } \\
\mathbf{x}: \text { Did not }\end{array}$ & \multicolumn{4}{|c|}{$\begin{array}{l}\text { Please check what you ate at } \\
\text { breakfast, lunch, and dinner. Also, } \\
\text { please check if you eat dairy } \\
\text { products and fruit. }\end{array}$} & $\begin{array}{l}\text { Sum of } \\
\text { the } \\
\text { check }\end{array}$ \\
\hline \multirow{3}{*}{ Example } & \multirow{3}{*}{$\begin{array}{l}5800 \\
\text { steps }\end{array}$} & & & \multirow{3}{*}{$x$} & \multirow{3}{*}{2} & \multirow{3}{*}{$\mathbf{x}$} & Grain & Grain & Srain & \multirow{2}{*}{ Mrik } & \multirow[b]{3}{*}{$/ 11$} \\
\hline & & & & & & & Vegetable & Vegotable & Vergetable & & \\
\hline & & & & & & & Fish/Meat & Fish \Kleat & FisK/Meat & Fruits & \\
\hline \multirow{3}{*}{ 6/11 Monday } & \multirow[b]{3}{*}{ steps } & & & & & & Grain & Grain & Grain & \multirow{2}{*}{ Milk } & \multirow[b]{3}{*}{$/ 11$} \\
\hline & & & & & & & Vegetable & Vegetable & Vegetable & & \\
\hline & & & & & & & Fish/Meat & Fish/Meat & Fish/Meat & Fruits & \\
\hline \multirow{3}{*}{ 6/12 Tuesday } & \multirow[b]{3}{*}{ steps } & & & & & & Grain & Grain & Grain & \multirow{2}{*}{ Milk } & \multirow[b]{3}{*}{$/ 11$} \\
\hline & & & & & & & Vegetable & Vegetable & Vegetable & & \\
\hline & & & & & & & Fish/Meat & Fish/Meat & Fish/Meat & Fruits & \\
\hline \multirow{3}{*}{ 6/13 Wednesday } & \multirow[b]{3}{*}{ steps } & & & & & & Grain & Grain & Grain & \multirow{2}{*}{ Milk } & \multirow[b]{3}{*}{$/ 11$} \\
\hline & & & & & & & Vegetable & Vegetable & Vegetable & & \\
\hline & & & & & & & Fish/Meat & Fish/Meat & Fish/Meat & Fruits & \\
\hline \multirow{3}{*}{ 6/14 Thursday } & \multirow[b]{3}{*}{ steps } & & & & & & Grain & Grain & Grain & \multirow{2}{*}{ Milk } & \multirow[b]{3}{*}{$/ 11$} \\
\hline & & & & & & & Vegetable & Vegetable & Vegetable & & \\
\hline & & & & & & & Fish/Meat & Fish/Meat & Fish/Meat & Fruits & \\
\hline & & & & & & & Grain & Grain & Grain & Milk & \\
\hline 6/15 Friday & & & & & & & Vegetable & Vegetable & Vegetable & & \\
\hline & steps & & & & & & Fish/Meat & Fish/Meat & Fish/Meat & Fruits & $/ 11$ \\
\hline & & & & & & & Grain & Grain & Grain & Milk & \\
\hline 6/16 Saturday & & & & & & & Vegetable & Vegetable & Vegetable & & \\
\hline & steps & & & & & & Fish/Meat & Fish/Meat & Fish/Meat & Fruits & $/ 11$ \\
\hline & & & & & & & Grain & Grain & Grain & Milk & \\
\hline 6/17 Sunday & & & & & & & Vegetable & Vegetable & Vegetable & & \\
\hline & steps & & & & & & Fish/Meat & Fish/Meat & Fish/Meat & Fruits & $/ 11$ \\
\hline [Comments to resea & chers] & & & & & & & & & & \\
\hline [Reply from researcl & & & & & & & & & & & \\
\hline
\end{tabular}

Figure S4 Daily-based physical activity, exercise, oral care, diet, and nutrition log. 


\section{References}

1. Watanabe Y, Tanimoto M, Oba N, Sanada K, Miyachi M, Ishii N. Effect of resistance training using bodyweight in the elderly: comparison of resistance exercise movement between slow and normal speed movement. Geriatr Gerontol Int. 2015;15(12):1270-1277.

2. Watanabe Y, Tanimoto M, Ohgane A, Sanada K, Miyachi M, Ishii N. Increased muscle size and strength from slow-movement, low-intensity resistance exercise and tonic force generation. J Aging Phys Act. 2013;21(1):71-84.

3. Murakami H, Tripette J, Kawakami R, Miyachi M. “Add 10 min for your health": the new Japanese recommendation for physical activity based on dose-response analysis. J Am Coll Cardiol. 2015;65(11):1153-1154.

4. Miyachi M, Tripette J, Kawakami R, Murakami H. "+10 min of physical activity per day": Japan is looking for efficient but feasible recommendations for its population. J Nutr Sci Vitaminol. 2015;61(Supp1):S7-S9.
5. Ministry of Health, Labour and Welfare. Physical Activity References for Health Promotion 2013. Available from: http://www.nibiohn.go.jp/ eiken/info/pdf/active2013-e.pdf. Accessed April 25, 2018.

6. Ministry of Health, Labour and Welfare and Ministry of Agriculture, Forestry and Fisheries. Japanese Food Guide Spinning Top. 2005. Available from: http://www.maff.go.jp/j/balance_guide/b_use/pdf/eng_reiari.pdf. Accessed April 25, 2018.

7. Yoshiike N, Hayashi F, Takemi Y, Mizoguchi K, Seino F. A new food guide in Japan: the Japanese food guide Spinning Top. Nutr Rev. 2007;65(4):149-154.

8. Oba S, Nagata C, Nakamura K, et al. Diet based on the Japanese Food Guide Spinning Top and subsequent mortality among men and women in a general Japanese population. J Am Diet Assoc. 2009;109(9):1540-1547.

9. Kurotani K, Akter S, Kashino I, et al. Quality of diet and mortality among Japanese men and women: Japan Public Health Center based prospective study. BMJ. 2016;352:11209.
Clinical Interventions in Aging

\section{Publish your work in this journal}

Clinical Interventions in Aging is an international, peer-reviewed journal focusing on evidence-based reports on the value or lack thereof of treatments intended to prevent or delay the onset of maladaptive correlates of aging in human beings. This journal is indexed on PubMed Central, MedLine,

\section{Dovepress}

CAS, Scopus and the Elsevier Bibliographic databases. The manuscript management system is completely online and includes a very quick and fair peer-review system, which is all easy to use. Visit http://www.dovepress. com/testimonials.php to read real quotes from published authors. 